\title{
Conception, réalisation et utilisation d'un simulateur de satellite dans une station deux voies de comparaison d'horloges par satellite
}

\section{Design, realization and application of a satellite simulator in a two-way satellite time and frequency transfer station}

\author{
Joseph ACHKAR
}

LNE-SYRTE, Observatoire de Paris, 61 Avenue de l’Observatoire, 75014 Paris, France, joseph.achkar@obspm.fr

\section{Résumé}

Le laboratoire national de métrologie pour le domaine tempsfréquences LNE-SYRTE a développé un simulateur de satellite microonde afin de déterminer le retard différentiel (et sa variation dans le temps) dans sa station terrienne deux voies. Un tel dispositif est de plus en plus recommandé par le groupe de travail TWSTFT du CCTF dans le but de mieux évaluer la variation du retard différentiel d'une station terrienne entre deux campagnes d'étalonnages consécutives. À côté de quelques rares simulateurs commercialement disponibles et basés sur une conception du VSL, nous avons développé un nouveau dispositif d'étalonnage avec au moins deux concepts différents de ceux déjà publiés dans la littérature : un processus plus rapide pour l'étalonnage des retards et une caractérisation de ses retards internes en utilisant un analyseur de réseau vectoriel micro-onde (ARVM). La partie clé de ce simulateur est l'utilisation d'un mélangeur micro-onde doublement équilibré et sa caractérisation en terme de retard absolu; celui-ci exige un système de mesure spécifique comme un ARVM permettant d'appliquer la technique de calibrage vectorielle par mélange en utilisant un ensemble «mélangeur/filtre » caractérisé en tant qu' «étalon » en liaison directe. Des mesures et incertitudes associées faites avec l'ARVM sont décrites dans ce papier. Elles sont complétées par des mesures effectuées avec le modem SATRE pendant des sessions d'étalonnage dédiées dans le but d'évaluer également les performances de l'ensemble du système en bruit (stabilité).

MOTS CLÉS : TWSTFT, MODEM SATRE, SIMULATEUR DE SATELLITE, COMPARAISON D'HORLOGES ATOMIQUES, ANALYSEUR DE RÉSEAU VECTORIEL, ÉTALONNAGE, STABILITÉ, RETARD, PARAMÈTRES S, BILAN D'INCERTITUDES.

\begin{abstract}
The French national metrology institute for time and frequency LNE-SYRTE has developed a new microwave satellite simulator to be used for the determination and monitoring of the delay difference in the Ku-band two-way satellite time and frequency transfer (TWSTFT) earth station. Such a device is more and more recommended by the CCTF working group on TWSTFT in order to better evaluate the ground station delay difference between two consecutive calibration campaigns. Besides few simulators commercially available and based on the VSL design, we have realized a new calibration device with at least two different concepts from those currently in use : a faster delay calibration process and a characterization of its internal delays using a microwave vector network analyzer (MVNA). The key component of this simulator is the use of a double-balanced microwave mixer and its characterization in terms of absolute delay; it requires a specific measurement system such as an MVNA allowing a vector mixer calibration technique with the use of a characterized mixer/filter as a through standard. The measurements and related uncertainties done with the MVNA are described in this paper. They are completed with those given by the SATRE modem during calibration sessions in order to perform the instability in noise of the whole system.
\end{abstract}

KEY WORDS: TWSTFT, SATRE MODEM, SATELLITE SIMULATOR, ATOMIC CLOCKS COMPARISON, VECTOR NETWORK ANALYZER, CALIBRATION, STABILITY, DELAY, S PARAMETERS, UNCERTAINTY BUDGET. 


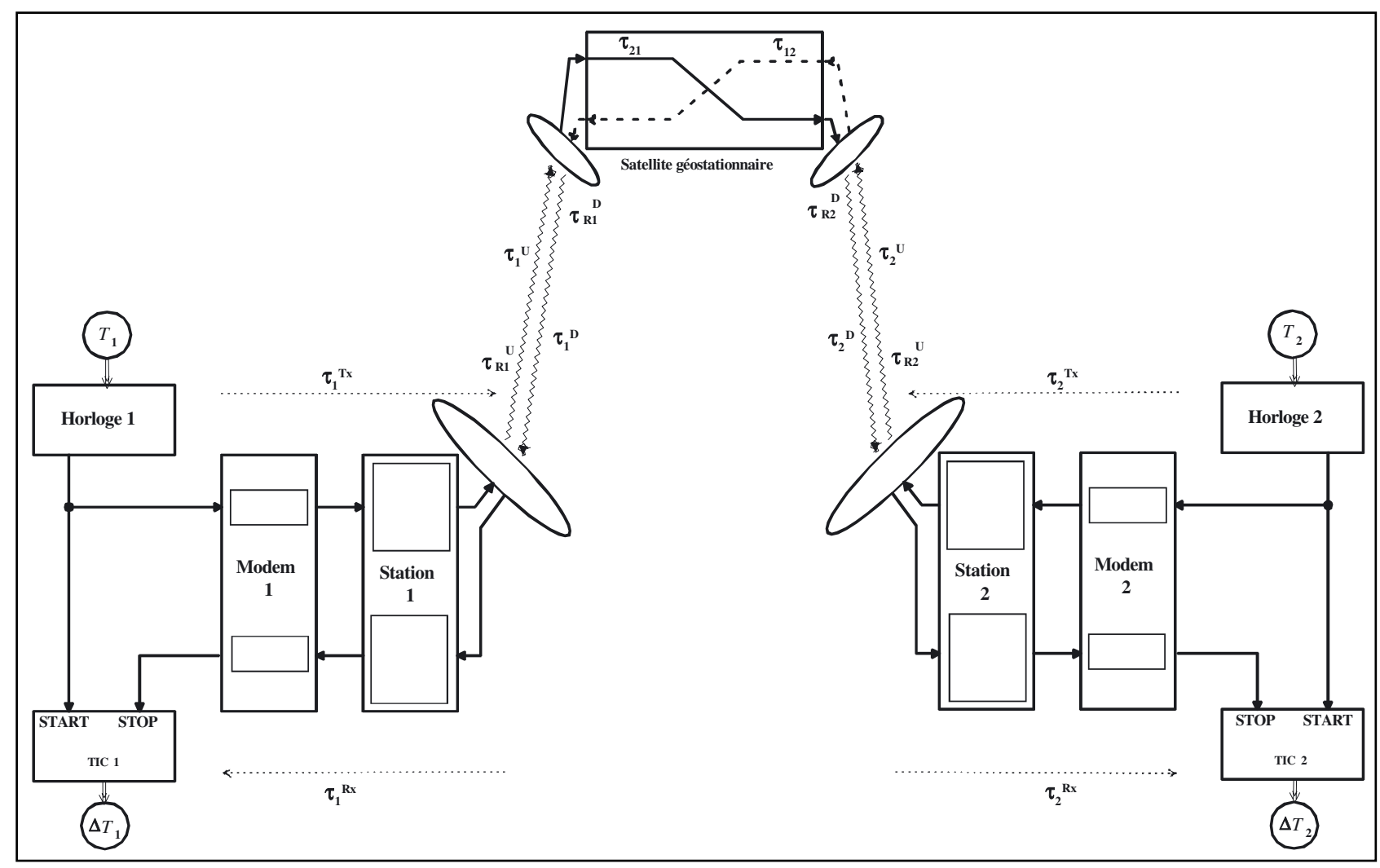

Fig. 1. - Principe de la technique TWSTFT.

\section{Acronymes utilisés}

ARVM Analyseur de Réseau Vectoriel Micro-ondes

BF Basse Fréquence

BIPM Bureau international des poids et mesures

CCD Common Clock Deviation (écart de mesure en colocation)

CCTF Comité Consultatif du Temps et des Fréquences

CNRS Centre national de la recherche ccientifique

FI Fréquence Intermédiaire $70 \mathrm{MHz}$

$\mathrm{Ku}$ bande de fréquence non normalisée, en usage dans les télécommunications par satellites pour désigner la bande $10 \mathrm{GHz}-18 \mathrm{GHz}$

LNA Low Noise Amplifier (amplificateur à faible bruit)

LNE Laboratoire national de métrologie et d'essais

SYRTE SYstèmes de Références Temps Espace

MVNA Microwave Vector Network Analyzer

OL Oscillateur Local

OP Observatoire de Paris (sigle historiquement utilisé en métrologie dans les comparaisons et publications internationales pour désigner le LNE-SYRTE)

OP01 Station terrienne deux voies utilisée sur les réseaux européens et américains

OP02 Station terrienne deux voies utilisée sur le réseau asiatique

PTB Physikalisch-Technische Bundesanstalt

pps pulse per second

R 120 désignation de bride de guide d'ondes (10 GHz-15 GHz) selon la norme de la Commission électrotechnique internationale (CEI)

RF Radiofréquence ou Haute Fréquence

SATRE SAtellite Time and Ranging Equipment

$\begin{array}{ll}\text { SOLT } & \text { Short }- \text { Open - Load - Through } \\ \text { SSPA } & \begin{array}{l}\text { Solid State Power Amplifier (amplificateur de puissance à } \\ \text { état solide) }\end{array} \\ \text { TAI } & \text { Temps Atomique International } \\ \text { TDA } & \text { Time Domain Analysis } \\ \text { TIC } & \text { Time Interval Counter } \\ \text { TRL } & \text { Through - Reflect - Line } \\ \text { TUG } & \text { Technische Universität Graz } \\ \text { TWSTFT } & \text { Two-Way Satellite Time and Frequency Transfer } \\ \text { UPMC } & \text { Université Pierre et Marie Curie (Paris 6) } \\ \text { UTC } & \text { Coordinated Universal Time (temps universel coordonné) } \\ \text { UTC(OP) } & \text { Temps Universel Coordonné de l'Observatoire de Paris } \\ \text { VSL } & \text { Van Swinden Laboratorium }\end{array}$

\section{Introduction}

La technique TWSTFT ([1-5]) est utilisée, parmi d'autres techniques, pour comparer des horloges atomiques distantes situées dans différents laboratoires de temps dans le monde (Europe, Amérique, Asie) contribuant au calcul de la référence internationale de temps, TAI, effectué par le BIPM. Les signaux d'horloges de deux stations terriennes distantes, situées à deux endroits géographiques distincts 1 et 2 (Fig. 1), sont transmis simultanément à travers un satellite géostationnaire, puis reçus de part et d'autre par ces mêmes stations. Chaque station mesure, à l'aide d'un compteur d'intervalles de temps, le retard entre le 1 pps du signal transmis et le 1 pps du signal reçu, soit $\Delta T_{1}$ pour la station 1 et $\Delta T_{2}$ pour la station 2 . 
L'écart de temps, $T_{1}-T_{2}$, entre les deux horloges atomiques est donné par (1) :

$$
T_{1}-T_{2}=\frac{1}{2}\left(\Delta T_{1}-\Delta T_{2}\right)+\frac{1}{2} \Delta \tau,
$$

où :

$$
\Delta \tau=\Delta \tau_{\mathrm{UD}}+\Delta \tau_{\mathrm{SAT}}+\Delta \tau_{\mathrm{R}}+\Delta \tau_{\mathrm{TR}}
$$

La technique TWSTFT permet d'éliminer tous les effets du premier ordre dus à la réciprocité des trajets des signaux. Le paramètre $\Delta \tau$ représente les effets résiduels non réciproques à déterminer. Il comporte les retards différentiels dus :

i) à la propagation atmosphérique pour les liaisons montante (U) et descendante (D) $\left(\Delta \tau_{\mathrm{UD}}\right)$;

ii) aux trajets dans le répéteur du satellite géostationnaire $\left(\Delta \tau_{\mathrm{SAT}}\right)$;

iii) à la rotation de la terre (effet Sagnac : $\left.\Delta \tau_{R}\right)$;

iv) et dans chaque station terrienne incluant les trajets $(\tau)$ d'émission (T) et de réception $(\mathrm{R})\left(\Delta \tau_{\mathrm{TR}}\right)$.

Ce dernier paramètre (3), développé ci-après, constitue la plus importante source d'erreur:

$$
\Delta \tau_{\mathrm{TR}}=\left[\left(\tau_{1}^{\mathrm{Tx}}-\tau_{1}^{\mathrm{Rx}}\right)-\left(\tau_{2}^{\mathrm{Tx}}-\tau_{2}^{\mathrm{Rx}}\right)\right] .
$$

Les retards différentiels, comme $\left(\tau_{1}^{\mathrm{Tx}}-\tau_{1}^{\mathrm{Rx}}\right)$ pour la station 1, sont à déterminer pour toutes les stations en comparaison, etc. Parmi les méthodes d'étalonnage qui peuvent exister, deux méthodes sont fortement recommandées par le groupe de travail TWSTFT du CCTF : étalonnage en relatif par station portable (méthode couramment appliquée) et étalonnage en absolu à l'aide de simulateurs de satellite (méthode en cours de développement).

Le laboratoire a développé une méthode d'étalonnage originale utilisant la technique du simulateur de satellite associée d'une procédure de mesure précise qui tient compte de tous les retards, à la fois du simulateur de satellite et de la station terrienne. L'intérêt de développer (ou de posséder) un simulateur de satellite est de caractériser les retards absolus d'une station terrienne et surtout de connaître leur variation dans le temps dans le but d'améliorer les incertitudes en transfert de temps. L'avantage de la méthode mise en oeuvre permet de réduire les incertitudes de mesures de ces retards.

\section{Dispositif d'étalonnage avec un simulateur de satellite}

Le dispositif d'étalonnage comprend, à côté de la station terrienne, un simulateur de satellite. La configuration de l'ensemble est présentée par la figure 2.

La station terrienne est composée principalement de deux ensembles : un ensemble interne, incluant le modem et un compteur d'intervalles de temps, installé dans

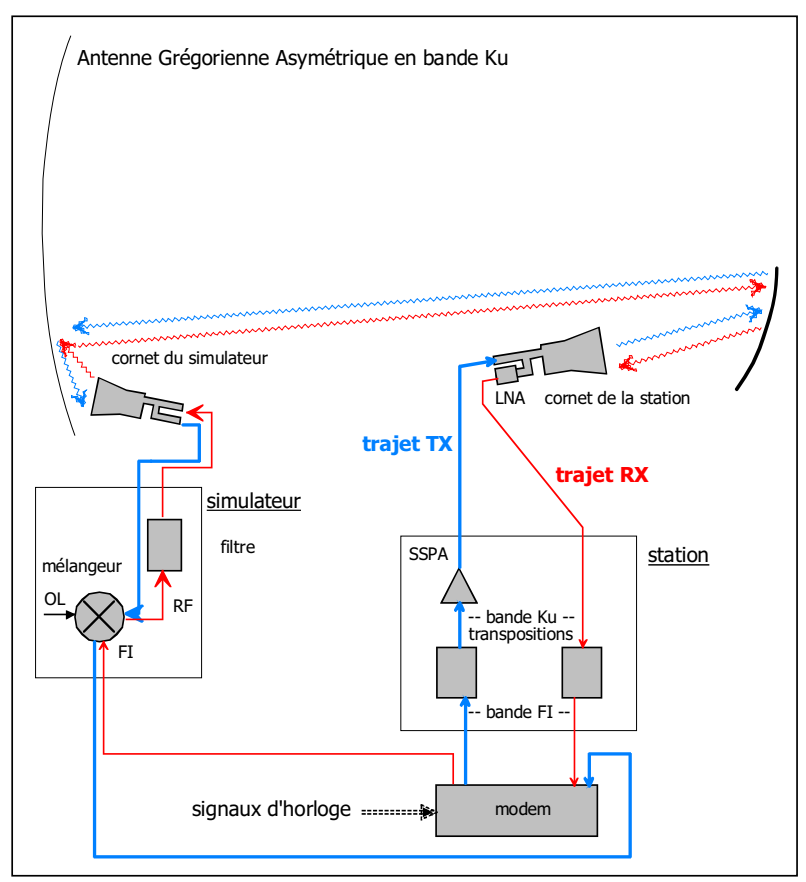

Fig. 2. - Implantation du simulateur de satellite dans la station deux voies du LNE-SYRTE.

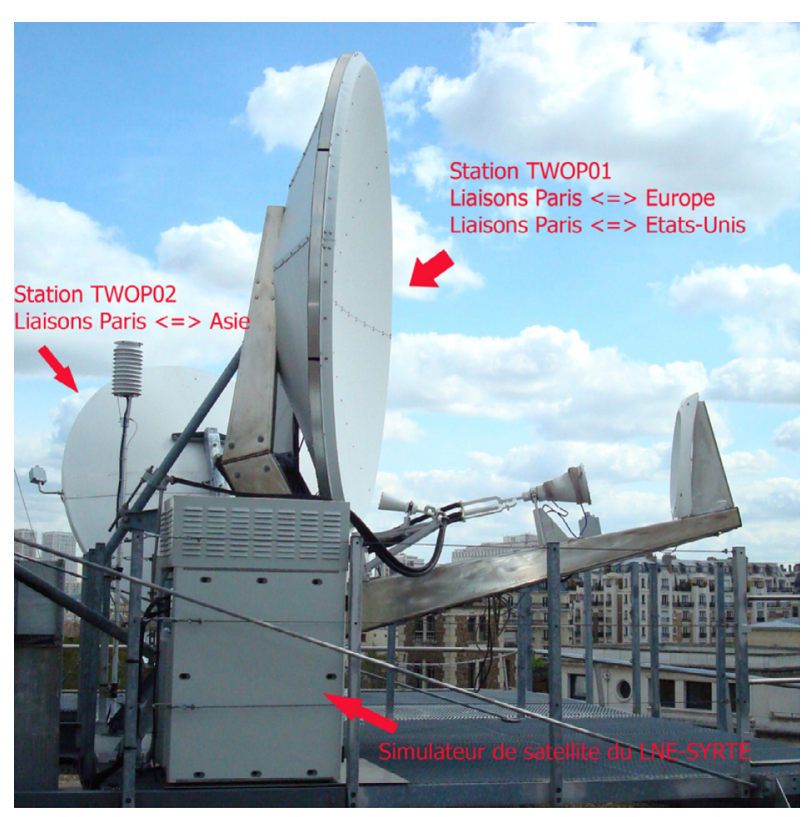

Fig. 3. - Vue d'ensemble, en extérieur, des 2 stations deux voies et du simulateur de satellite.

une salle régulée en température à $22,5^{\circ} \mathrm{C} \pm 0,5^{\circ} \mathrm{C}$; et un ensemble externe, comportant les convertisseurs haute fréquence et basse fréquence et une antenne asymétrique à double réflecteur, installé sur la terrasse d'un bâtiment de l'Observatoire de Paris (Fig. 3).

Le simulateur est composé de :

- d'un oscillateur local micro-ondes piloté par une référence de fréquence externe (ici c'est un des masers à hydrogène actif du laboratoire); 
- d'un cornet à excitation rectangulaire (R120) et à ouverture circulaire avec une double polarisation linéaire orthogonale;

- deux guides d'ondes souples et deux adaptateurs ;

- d'un mélangeur micro-ondes équilibré ;

- un filtre BF;

- de quatre commutateurs électromécaniques, des câbles coaxiaux et des connecteurs [5].

Le simulateur est conçu [6,7] pour une utilisation partielle en extérieur (guides souples, cornet), les autres équipements sont installés dans une enceinte régulée en température $(25 \pm 5){ }^{\circ} \mathrm{C}$ avec une humidité relative inférieure à $50 \%$.

\section{Description de la méthode de mesure}

D'après la conception du simulateur dessiné au laboratoire, la caractérisation du retard différentiel des deux trajets d'émission et de réception relatifs à la station terrienne repose sur les deux mesures successives suivantes :

La première configuration illustrée dans la figure 4 (tracés plein et discontinu entre les cornets) est considérée dans la mesure du retard global, en mode de transmission par la station, soit $\delta t_{1}=\tau_{1}^{\mathrm{Tx}}+C a l_{1}$, où :

$$
\tau_{1}^{\mathrm{Tx}}=d_{\text {ModemTx }}+d_{\mathrm{S} 1}+d_{\mathrm{C} 1}+d_{\mathrm{UC}}+d_{\mathrm{C} 2}+d_{\mathrm{HstaTx}}
$$

$\tau_{1}^{\mathrm{Tx}}$ représente le retard du trajet d'émission dans la station terrienne, incluant la partie interne de transmission du modem, et :

$$
\begin{aligned}
\mathrm{Cal}_{1}= & d_{\mathrm{R} 1}+d_{\mathrm{HsimRx}}+d_{\mathrm{C} 3}+d_{\mathrm{A} 1}+d_{\mathrm{C} 4}+d_{\mathrm{S} 4}+d_{\mathrm{Mix} 1} \\
& +d_{\mathrm{S} 3}+d_{\mathrm{FL} 2}+d_{\mathrm{S} 2}+d_{\mathrm{ModemRx}}
\end{aligned}
$$

$\mathrm{Cal}_{1}$ représente l'ensemble des retards couvrant une partie du simulateur de satellite et incluant la partie interne de réception du modem.

La deuxième configuration illustrée dans la figure 4 (tracés rouge et noir) est considérée dans la mesure du retard global, en mode de réception par la station, soit $\delta t_{2}=\tau_{1}^{\mathrm{Rx}}+\mathrm{Cal}_{2}$, où :

$$
\tau_{1}^{\mathrm{Rx}}=d_{\mathrm{HstaRx}}+d_{\mathrm{C} 7}+d_{\mathrm{DC}}+d_{\mathrm{C} 8}+d_{\mathrm{S} 2}+d_{\text {ModemRx }}
$$

$\tau_{1}^{\mathrm{Rx}}$ représente le retard du trajet de réception dans la station terrienne, incluant la partie interne de réception du modem, et :

$$
\begin{aligned}
\mathrm{Cal}_{2}= & d_{\mathrm{ModemTx}}+d_{\mathrm{S} 1}+d_{\mathrm{C} 5}+d_{\mathrm{S} 3}+d_{\mathrm{Mix} 2}+d_{\mathrm{S} 4} \\
& +d_{\mathrm{FL} 1}+d_{\mathrm{A} 2}+d_{\mathrm{C} 6}+d_{\mathrm{HsimTx}}+d_{\mathrm{R} 2}
\end{aligned}
$$

$\mathrm{Cal}_{2}$ représente l'ensemble des retards couvrant une partie du simulateur de satellite et incluant la partie interne de transmission du modem.

Ainsi, en différenciant les deux mesures, on obtient le retard recherché $\tau_{1}^{\mathrm{Tx}}-\tau_{1}^{\mathrm{Rx}}=\delta t_{1}-\delta t_{2}-\left(\mathrm{Cal}_{1}-\mathrm{Cal}_{2}\right)$. Les paramètres $\mathrm{Cal}_{1}$ et $\mathrm{Cal}_{2}$ sont déterminés de façon exacte en mesurant les retards de tous les composants constituant le simulateur, à l'aide d'un compteur d'intervalles de temps et d'un analyseur de réseau vectoriel [8].

\section{Caractérisation du dispositif (stabilité)}

Le bruit de mesure, dans le domaine fréquentiel, de l'ensemble du dispositif, comprenant la station deux voies et le simulateur de satellite, est analysé en premier. Le retard temporel est mesuré sur le long terme, sur une période d'une vingtaine de jours, à raison de douze sessions de mesure par jour (toutes les deux heures) enregistrant $2 \times 120$ points de mesure (une donnée par seconde) à chaque session. Le rapport signal (porteuse) à bruit obtenu est de l'ordre de $68 \mathrm{dBHz}$ (excellent), pour toute la durée des mesures.

Les fluctuations de la puissance mesurée, en réception, pour les trajets Tx (émission par la station) et Rx (émission par le simulateur) restent inférieures à $1 \mathrm{~dB}$, sur la période totale de mesure, d'après $[9,10]$. L'écart temporel d'Allan correspondant, lié au retard différentiel $0,5 \times(\mathrm{Tx}-\mathrm{Rx})$ du dispositif, est représenté sur la figure 5 .

Un écart temporel inférieur à 50 ps est maintenu entre $0,3 \mathrm{~d}$ et $3 \mathrm{~d}$, résultat particulièrement satisfaisant qui permet une caractérisation rigoureuse de la station deux voies, étalonnée, par ailleurs, avec une incertitude combinée de l'ordre de 1 ns [11].

De plus, et dans le but de déterminer le bruit résiduel à court terme de l'ensemble du dispositif, une expérience particulière est menée toutes les $1 \mathrm{~s}$, de façon continue, sur une période d'analyse allant de $1 \mathrm{~h}$ à $3 \mathrm{~h}$. En plus des deux configurations appliquées au simulateur, une troisième configuration est considérée ici en supprimant, dans le montage, la partie rayonnée par les cornets. Afin d'effectuer cette expérience, il a été nécessaire d'interrompre une ou deux sessions régulières de mesure.

Dans cette expérience, la caractérisation du dispositif est faite, avec et sans les cornets, pour pouvoir évaluer l'effet de la propagation micro-onde rayonnée, en terme de bruit de mesure. D'après la figure 6, il est clairement montré, que cet effet est éliminé dans le trajet Tx, puisqu'il rejoint les performances d'un système fermé non rayonné (< 10 ps à 1 s), alors qu'il dépasse légèrement les 10 ps pour le trajet Rx. Les résultats obtenus montrent un excellent bruit de mesure du dispositif, et donc de la station deux voies du laboratoire. Au-delà des performances atteintes par l'ensemble du dispositif, cette expérience a été d'une aide précieuse dans l'optimisation du positionnement du cornet du simulateur, dans l'environnement électromagnétique de la parabole.

\section{Caractérisation du dispositif (retard)}

La conception du simulateur de satellite du laboratoire et la méthode appliquée nécessitent la connaissance au préalable de ses retards internes. Ceux-ci peuvent être déterminés en utilisant une technique de très haute performance fournie par un analyseur de réseau vectoriel micro-ondes approprié.

L'équation traduisant la relation entre le retard différentiel de la station terrienne et les retards internes du 


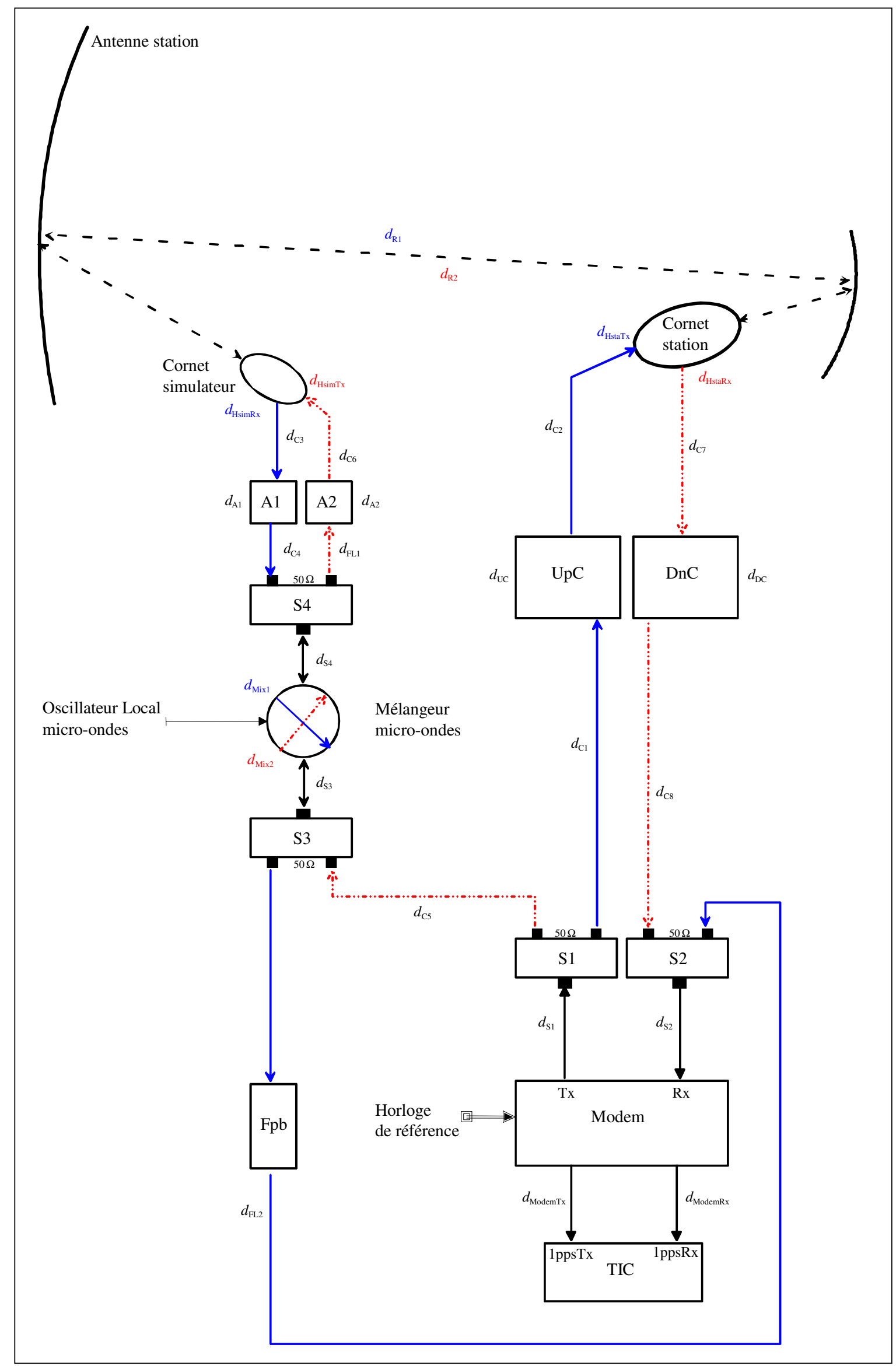

Fig. 4. - Tracé du trajet total en émission (trait plein) et en réception (trait pointillé) et identification des différents retards ( $d . .$.$) .$ 


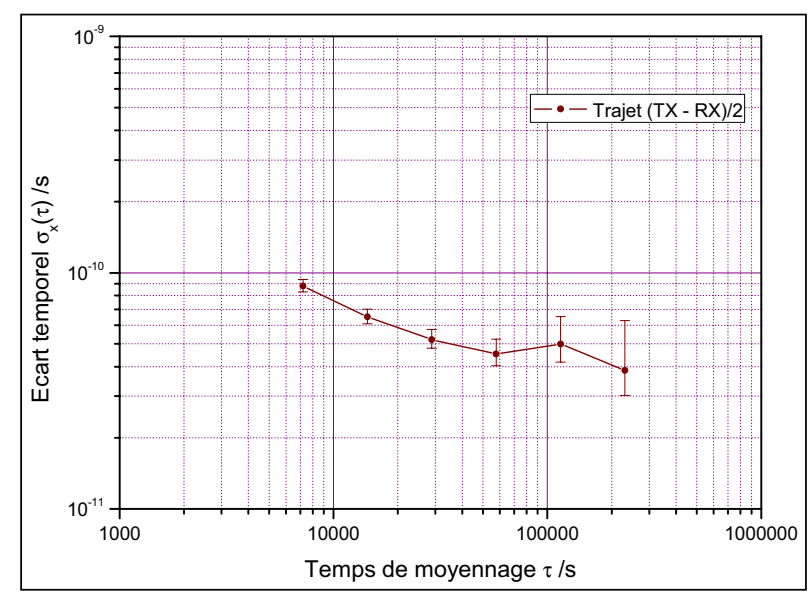

Fig. 5. - Stabilité de temps du retard différentiel du dispositif.

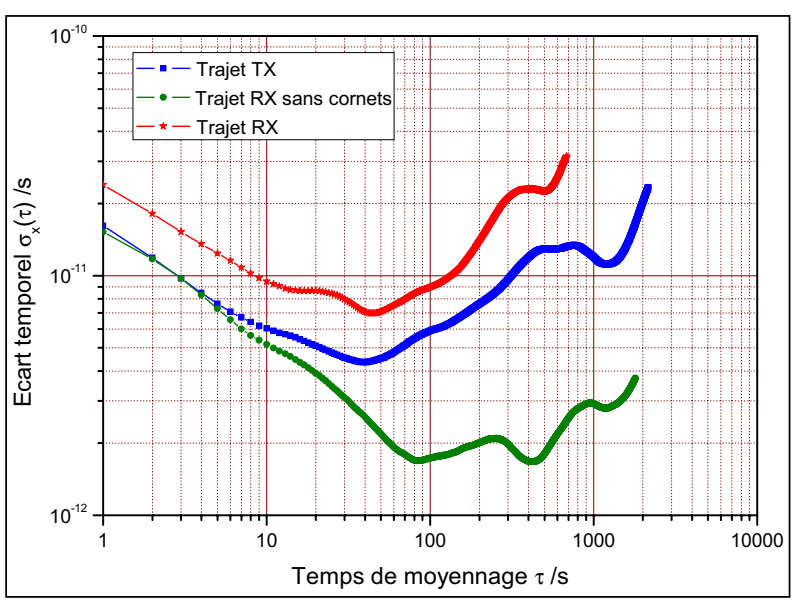

Fig. 6. - Stabilité de temps des retards des trajets Tx et Rx, avec et sans les cornets d'antenne.

simulateur de satellite est donnée par (d'après la section 3) :

$$
\tau_{1}^{\mathrm{Tx}}-\tau_{1}^{\mathrm{Rx}}=\delta t_{1}-\delta t_{2}-\left(C a l_{1}-C a l_{2}\right) .
$$

Après développement, le paramètre $\mathrm{Cal}=\mathrm{Cal}_{1}-\mathrm{Cal}_{2}$ peut être exprimé comme :

$$
\text { Cal }=\left[d_{\text {Modem, Rx }}-d_{\text {Modem, Tx }}\right]+C a l_{0} .
$$

Le paramètre $\left[d_{\text {Modem, Rx }}-d_{\text {Modem,Tx }}\right]$ est mesuré par un oscilloscope numérique, tandis que le paramètre $\mathrm{Cal}_{0}$ est déterminé à l'aide d'un ARVM. Ce dernier paramètre comprend tous les retards internes du simulateur de satellite, y compris le retard du trajet entre les deux cornets :

$$
\begin{aligned}
& \mathrm{Cal}_{0}=\left(d_{\mathrm{S} 2}-d_{\mathrm{S} 1}\right)+\left(d_{\mathrm{S} 3}-d_{\mathrm{S} 3}\right)+\left(d_{\mathrm{S} 4}-d_{\mathrm{S} 4}\right) \\
& +\left(d_{\mathrm{FL} 2}-d_{\mathrm{FL} 1}\right)+\left(d_{\mathrm{Mix} 1}-d_{\mathrm{Mix} 2}\right)+\left(d_{\mathrm{C} 4}-d_{\mathrm{C} 5}\right) \\
& +\left[\left(d_{\mathrm{A} 1}+d_{\mathrm{C} 3}\right)-\left(d_{\mathrm{A} 2}+d_{\mathrm{C} 6}\right)\right]+\left(d_{\mathrm{HsimRx}}-d_{\mathrm{HsimTx}}\right) \\
& +\left(d_{\mathrm{R} 1}-d_{\mathrm{R} 2}\right)
\end{aligned}
$$

D'après la figure 4 :

$\left(d_{\mathrm{S} 2}-d_{\mathrm{S} 1}\right):$ retard différentiel des commutateurs à la fréquence FI ;

$\left(d_{\mathrm{S} 3}-d_{\mathrm{S} 3}\right)$ : retard différentiel des deux accès du même commutateur à la fréquence FI ;
$\left(d_{\mathrm{S} 4}-d_{\mathrm{S} 4}\right):$ retard différentiel des deux accès du même commutateur en bande $\mathrm{Ku}$;

$\left(d_{\mathrm{FL} 2}-d_{\mathrm{FL} 1}\right):$ retard différentiel de deux liaisons coaxiales, incluant un filtre, à $70 \mathrm{MHz}$ et en bande $\mathrm{Ku}$;

$\left(d_{\mathrm{Mix} 1}-d_{\mathrm{Mix} 2}\right):$ retard différentiel du mélangeur, sur ces deux accès RF et FI;

$\left(d_{\mathrm{C} 4}-d_{\mathrm{C} 5}\right):$ retard différentiel de deux liaisons coaxiales, à $70 \mathrm{MHz}$ et en bande $\mathrm{Ku}$;

$\left(d_{\mathrm{A} 1}+d_{\mathrm{C} 3}\right)-\left(d_{\mathrm{A} 2}+d_{\mathrm{C} 6}\right):$ retard différentiel de quatre liaisons guidées et coaxiales, en bande $\mathrm{Ku}$;

$\left(d_{\mathrm{HsimRx}}-d_{\mathrm{HsimTx}}\right):$ retard différentiel du cornet du simulateur, en bande $\mathrm{Ku}$;

$\left(d_{\mathrm{R} 1}-d_{\mathrm{R} 2}\right)$ : retard différentiel du trajet de propagation rayonnée entre les deux cornets, en bande $\mathrm{Ku}$.

\subsection{Caractérisation des composants linéaires du simulateur}

L'ARVM Agilent PNA E8362C du laboratoire est utilisé. Différentes méthodes de calibrage et de mesure sont appliquées $([12,13])$ en fonction des bandes de fréquences d'opération et des modes de mesure considérés :

- La méthode TRL appliquée dans la bande de fréquence $10 \mathrm{GHz}-15 \mathrm{GHz}$, en utilisant les kits de calibrage, Agilent 85052C et Flann 17720, munis de connecteurs de précision en $3,5 \mathrm{~mm}$ et de brides rectangulaires en R 120, respectivement;

- La méthode SOLT appliquée dans la bande de fréquence $50 \mathrm{MHz}-90 \mathrm{MHz}$, en utilisant le kit de calibrage Agilent $85052 \mathrm{C}$ muni de connecteurs de précision en $3,5 \mathrm{~mm}$;

- La méthode temporelle TDA dans le cas où des mesures vectorielles en réflexion sont effectuées.

De plus, des kits électroniques de calibrage et mécaniques de vérification, des câbles et des adaptateurs de précision sont également utilisés selon le cas, en calibrage et en mesure.

Les mesures concernent l'ensemble des éléments constituant le simulateur de satellite (câbles coaxiaux, commutateurs, adaptateurs, guides d'ondes, cornet, atténuateurs et filtres), et sont basées sur des mesures de paramètres de dispersion $S$, en réflexion et en transmission pour le module et la phase, dans les deux modes de configuration (transmission et réception) du dispositif (Fig. 4). À titre d'exemple, des résultats numériques relatifs aux différents retards sont présentés dans la section 5.3.

\subsection{Retard absolu du mélangeur micro-onde}

Par construction, l'ARVM effectue des mesures de composants linéaires en fonction de la fréquence. Lorsque des composants non linéaires sont à caractériser (par exemple, le mélangeur installé dans le simulateur de satellite), un concept différent de mesure est nécessaire à appliquer sur l'ARVM (si seulement l'analyseur 


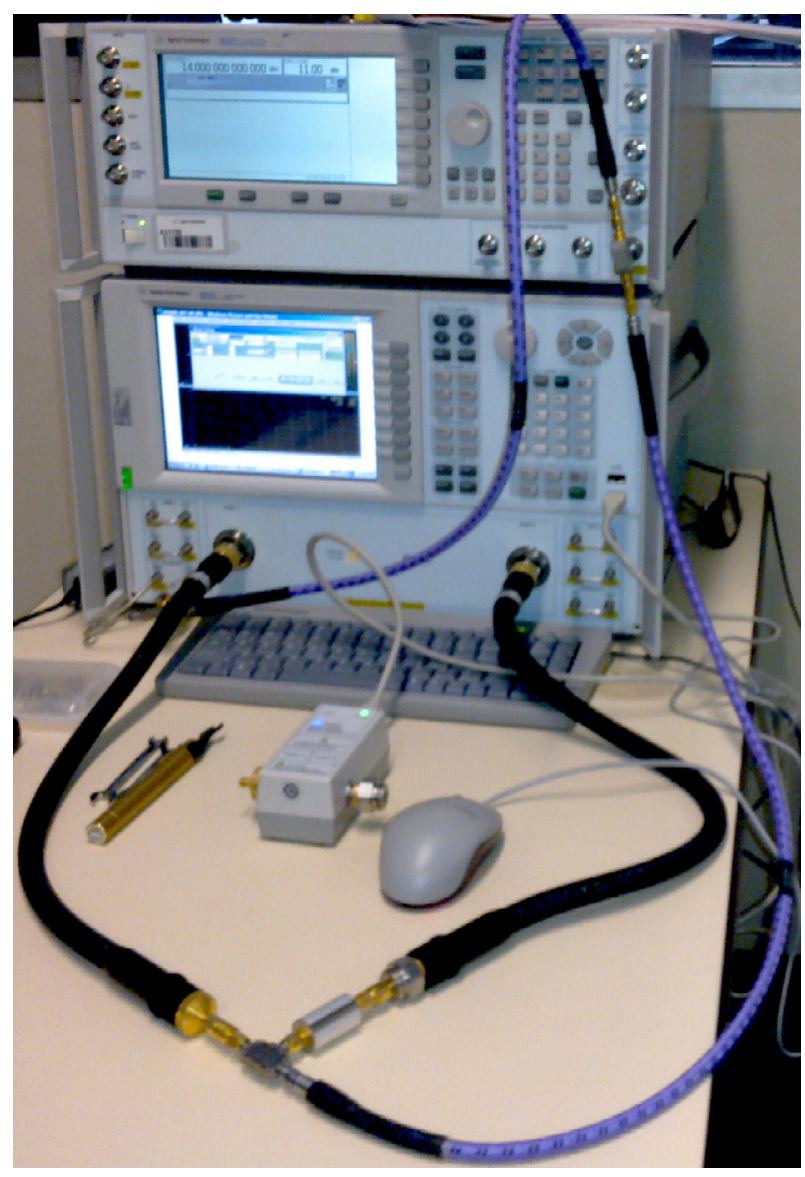

Fig. 7. - Banc de mesure du retard absolu d'un mélangeur micro-ondes.

en est équipé) entraînant un changement de fréquence, entre l'entrée et la sortie du dispositif sous test.

Avec le soutien technique et matériel d'Agilent Technologies France, un nouveau processus a été appliqué qui permet de fournir une solution de mesure par correction vectorielle des paramètres de mesure du mélangeur, les fréquences FI (OL) et RF fonctionnant en mode de balayage de fréquence. Cette technique de mesure utilise une instrumentation d'analyse de réseau adaptée avec une possibilité de changement de fréquence, corrigée vectoriellement à l'aide d'une méthode de calibrage par mélange [14].

Le montage spécifique du banc de mesure du retard absolu du mélangeur est présenté dans la figure 7. Deux mélangeurs de référence sont utilisés dans cette expérience. Ils sont associés en sortie à des filtres passe-bas et passe-bande afin d'éliminer les produits d'intermodulation, gênants pour la mesure. Des câbles de grande qualité et un diviseur de puissance sont utilisés et des serrages appropriés sont effectués.

Les mesures sont effectuées en deux étapes, le composant sous test étant le mélangeur Miteq DM0416LW2 :

\section{Mesure en mode de transposition basse fréquence}

La mesure en mode de balayage de fréquence pour les deux signaux OL et RF, afin d'obtenir une valeur fixe

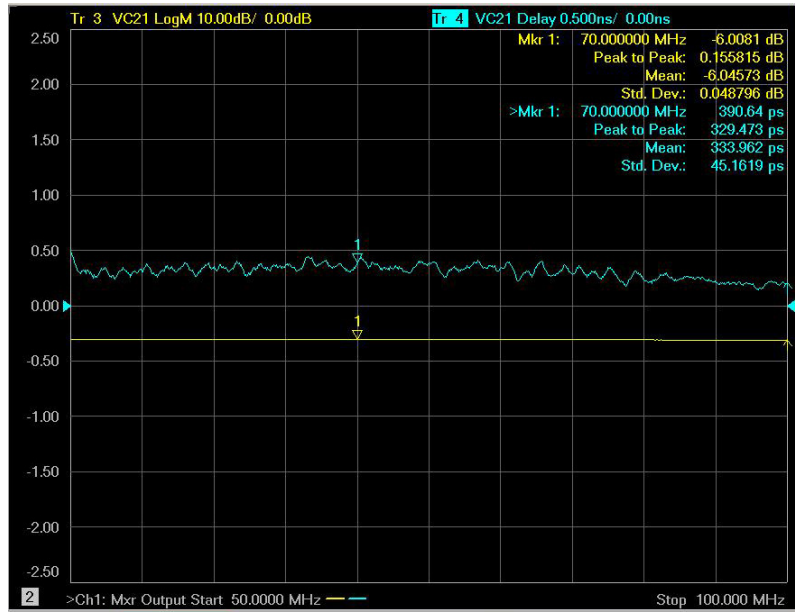

Fig. 8. - Mesure du retard absolu du mélangeur en mode de transposition basse fréquence.

FI est excessivement délicate. En effet, trois mesures sont effectuées simultanément à la fréquence d'opération et à +/-1 MHz de cette fréquence, dans les conditions de régime de fonctionnement linéaire du mélangeur [15]. Le retard absolu, exprimé en nanoseconde, est déterminé à partir de la différence de phase, exprimée en degré, sur une bande de fréquence de $2 \mathrm{MHz}$ (la fréquence étant elle-même exprimée en mégahertz), soit :

$$
\tau_{\mathrm{dn}, \text { mixer }}=\frac{\varphi_{F_{0}}\left(F_{0}+1\right)-\varphi_{F_{0}}\left(F_{0}-1\right)}{360 \times 2 \times 10^{6}} .
$$

Ici nous avons appliqué une autre méthode, plus efficace, qui permet d'afficher directement le résultat du retard de groupe à l'écran de l'ARVM. En effet, nous avons réalisé la mesure en mode de balayage de fréquence pour les deux signaux RF et FI, pour un signal OL à fréquence fixe. Dans cette expérience, deux filtres passe-bas sont associés aux voies FI des mélangeurs (de référence et de mesure). Ils coupent à partir de $1 \mathrm{GHz}$ et présentent des pertes d'insertion de $0,2 \mathrm{~dB}$ à $70 \mathrm{MHz}$. Les résultats de mesure sont reportés dans la figure 8 : pour $F_{0}=70 \mathrm{MHz}$, le retard absolu en mode de transposition basse fréquence vaut $\tau_{\mathrm{dn} \text {,mixer }}=d_{\mathrm{Mix} 1} \approx 334 \mathrm{ps} \pm 46$ ps et le gain de conversion mesuré est de $6 \mathrm{~dB}$.

\section{Mesure en mode de transposition haute fréquence}

La mesure dans ce sens est plus classique mais toujours aussi délicate car cette fois-ci elle se produit en haute fréquence et le retard absolu est directement affiché sur l'analyseur. Cette mesure permet de caractériser le mélangeur directement en termes de gain de conversion et de temps de propagation de groupe. Dans ce cas, les filtres à réjection de fréquence associés aux mélangeurs jouent un rôle prépondérant dans la mesure (indispensable sur la voie de référence, recommandé sur la voie de mesure). Étant donné l'indisponibilité de filtres à $12 \mathrm{GHz}$ pour cette expérience, nous avons effectué cette mesure à $10 \mathrm{GHz}$. Ainsi, un filtre à bande très étroite connecté sur la voie RF du mélangeur de référence est utilisé. Il possède une bande passante de $200 \mathrm{MHz}$ et des pertes d'insertion de $0,8 \mathrm{~dB}$ à $10 \mathrm{GHz}$. Les résultats de mesure sont 
Tableau 1

Valeurs numériques des retards différentiels de l'ensemble du dispositif.

\begin{tabular}{|c|c|c|}
\hline $\mathbf{N}^{\circ}$ paramètre & Identification du paramètre & Retard différentiel (ns) \\
\hline 1 & $d_{\mathrm{ModemRx}}-d_{\mathrm{ModemTx}}$ & 14062,9 \\
\hline 2 & $d_{\mathrm{S} 2}-d_{\mathrm{S} 1}(70 \mathrm{MHz})$ & 0,0 \\
\hline 3 & $d_{\mathrm{S} 3}-d_{\mathrm{S} 3}(70 \mathrm{MHz})$ & 0,0 \\
\hline 4 & $d_{\mathrm{S} 4}-d_{\mathrm{S} 4}($ bande $\mathrm{Ku})$ & 0,0 \\
\hline 5 & $d_{\mathrm{FL} 2}-d_{\mathrm{FL} 1}(\mathrm{FI}-$ bande $\mathrm{Ku})$ & 241,9 \\
\hline 6 & $d_{\mathrm{Mix} 1}-d_{\mathrm{Mix} 2}(\mathrm{Ku} / \mathrm{FI}-\mathrm{FI} / \mathrm{Ku})$ & 0,0 \\
\hline 7 & $d_{\mathrm{C} 4}-d_{\mathrm{C} 5}($ bande $\mathrm{Ku}-\mathrm{FI})$ & $-237,3$ \\
\hline 8 & $\left(d_{\mathrm{A} 1}+d_{\mathrm{C} 3}\right)-\left(d_{\mathrm{A} 2}+d_{\mathrm{C} 6}\right)($ bande Ku$)$ & $-0,8$ \\
\hline 9 & $d_{\mathrm{HsimRx}}-d_{\mathrm{HsimTx}}($ bande $\mathrm{Ku})$ & $-9,2$ \\
\hline 10 & $d_{\mathrm{R} 1}-d_{\mathrm{R} 2}($ bande $\mathrm{Ku})$ & 0,0 \\
\hline 11 & $C a l_{0}=C a l_{1}-C_{2}($ modem exclus $)$ & $-5,4$ \\
\hline 12 & $C a l=C a l_{1}-C a l_{2}($ modem inclus $)$ & 14057,5 \\
\hline 13 & $\delta t_{1}-\delta t_{2}\left(\mathrm{~d}^{\prime}\right.$ après $\left.[14]\right)$ & 24,0 \\
\hline $\mathbf{1 4}$ & $\tau_{\mathbf{1}}^{\mathbf{T x}}-\tau_{\mathbf{1}}^{\mathbf{R x}}(\mathbf{O P 0 1})$ & $\mathbf{- 1 4 0 3 3 , 5}$ \\
\hline
\end{tabular}

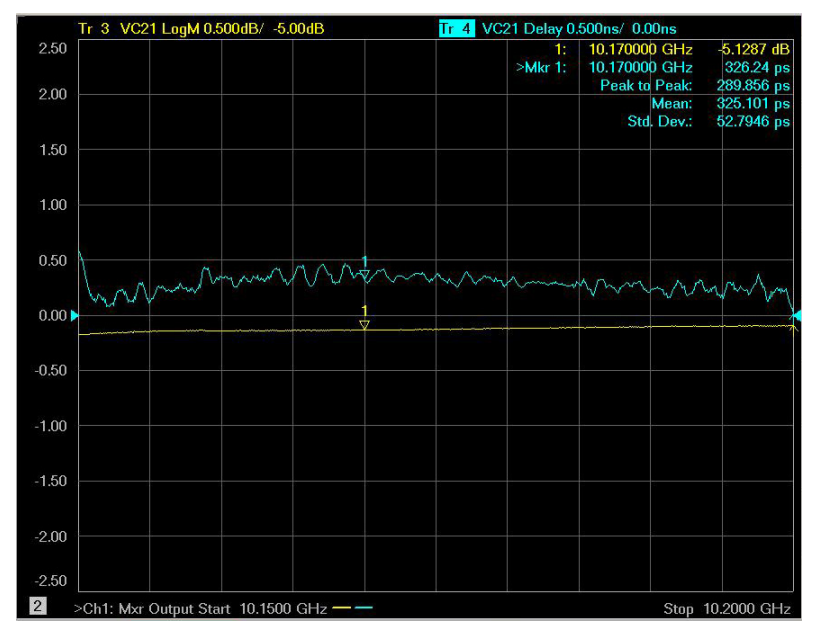

Fig. 9. - Mesure du retard absolu du mélangeur en mode de transposition haute fréquence.

reportés dans la figure 9 : pour $F_{0}=10,170000 \mathrm{MHz}$, le gain de conversion mesuré est d'environ $5,2 \mathrm{~dB}$ et le retard absolu en mode de transposition haute fréquence vaut $\tau_{\text {up,mixer }}=d_{\text {Mix } 2} \approx 325$ ps \pm 53 ps.

Ainsi les excellents premiers résultats obtenus montrent que le mélangeur micro-ondes utilisé est relativement réciproque en termes de retard de groupe. La mesure par correction scalaire des paramètres du mélangeur montre un écart de $0,5 \mathrm{~dB}$ sur le gain de conversion dans les deux sens, ce qui exclut une réciprocité totale du mélangeur.

On peut toutefois améliorer davantage (en adaptation et en dynamique) le montage de mesure en y rajoutant des atténuateurs côté source (port 1) et côté mesure (port 2) de l'ARVM.

\subsection{Exemple de détermination du retard différentiel de la station}

Le simulateur de satellite a été expérimenté avec la station OP01, sur une période de vingt jours, pendant les heures UTC impaires (en dehors du programme des sessions régulières deux voies). Les mesures sont effectuées, sur une base journalière de douze sessions de mesure, à raison de $2 \times 120$ données ( 1 s) de mesure par session, en utilisant le code 0 (code assigné à la station OP01 par le groupe de travail TWSTFT du CCTF) du modem SATRE à 2,5 Mchips/s. Le rapport signal (porteuse) à bruit $C / N_{0}$ reçu par la station, quelque soit le mode de transmission (par la station ou par le simulateur), était excellent $(68 \mathrm{dBHz})$

Les retards différentiels de l'ensemble des trajets identifiés dans le dispositif, sont reportés dans le tableau 1. Selon la configuration de chaque ensemble au sein du dispositif, les mesures de retard ont été relevées aux trois fréquences d'opération : $70 \mathrm{MHz}$, $12,518200 \mathrm{GHz}$ et 14,013200 GHz. Les différents paramètres sont identifiés et déterminés de la manière suivante :

- le paramètre $\mathrm{n}^{\circ} 1$, est déterminé par le modem SATRE;

- les paramètres n 2 à 9, sont déterminés à partir des mesures faites avec l'ARVM ;

- le paramètre $n^{\circ} 10$, est déterminé en considérant les trajets réciproques;

- les paramètres $n^{\circ} 11$ et 12 , sont calculés ;

- le paramètre $n^{\circ} 13$, est mesuré par le compteur d'intervalles de temps interne du modem;

- les paramètres n 14 à 18 sont calculés.

D'après le tableau 1 , le retard différentiel calculé de la station OP01 vaut :

$$
\tau_{1}^{\mathrm{Tx}}-\tau_{1}^{\mathrm{Rx}}(\mathrm{OP} 01)=-14033,50 \mathrm{~ns} \pm 0,40 \mathrm{~ns} .
$$

En tenant compte des résultats de la campagne d'étalonnage [11], on peut déduire le retard différentiel, soit de la station portable ayant servie dans la campagne soit, de la station distante ayant participée à cette campagne (exemple : TUG, VSL01, etc.). 
Tableau 2

Budget d'incertitudes de mesure de l'amplitude du paramètre $S_{21}(0,2 \mathrm{~dB})$.

\begin{tabular}{|l|c|c|c|c|}
\hline \multicolumn{1}{|c|}{ Contribution } & Incertitude $(\mathbf{d B})$ & Distribution & Division & Incertitude type (dB) \\
\hline $\mathrm{B}_{1}:$ linéarité du système & 0,0004 & Gaussienne & 2 & 0,0002 \\
\hline $\mathrm{B}_{2}:$ désadaptation & 0,0164 & Rectangulaire & 1,4142 & 0,0116 \\
\hline $\mathrm{B}_{3}:$ isolation & 0,0003 & Rectangulaire & 1,7321 & 0,0002 \\
\hline $\mathrm{B}_{4}:$ bruit du système & 0,0040 & Gaussienne & 2 & 0,0020 \\
\hline $\mathrm{B}_{5}:$ flexion du câble & 0,0100 & Gaussienne & 2 & 0,0050 \\
\hline $\mathrm{B}_{6}:$ conditions ambiantes & 0,0020 & Rectangulaire & 1,7321 & 0,0012 \\
\hline $\mathrm{A}_{1}:$ répétabilité du système & 0,0020 & Gaussienne & 2 & 0,0010 \\
\hline $\mathrm{A}_{2}:$ répétabilité du connecteur & 0,0200 & Gaussienne & 2 & 0,0100 \\
\hline Incertitude combinée, $\boldsymbol{u}\left|\boldsymbol{S}_{\mathbf{2 1}}\right|$ & & & & $\mathbf{0 , 0 1 6 3}$ \\
\hline
\end{tabular}

L'incertitude combinée sur le retard différentiel de la station OP01, calculé à partir des mesures effectuées par le modem et l'analyseur de réseau vectoriel, est de l'ordre de 400 ps. Un exemple détaillé du budget d'incertitudes pour les mesures effectuées à l'analyseur de réseau vectoriel est présenté dans la section 5.4.

\section{4. Évaluation des incertitudes de mesure à l'aide d'un analyseur de réseau vectoriel}

La caractérisation du simulateur de satellite développé au laboratoire, en termes de paramètres de dispersion et de retard de groupe, est faite à l'aide de l'analyseur de réseau vectoriel micro-onde. Deux cas sont considérés :

- éléments à deux accès : quel que soit le support d'excitation (sur ligne coaxiale ou sur guide d'ondes), une mesure vectorielle des paramètres de dispersion $S$ est effectuée, le retard de groupe étant déterminé à partir des mesures de phase en mode de transmission;

- éléments à un accès : le calibrage est effectué dans le domaine fréquentiel; le retard de groupe est déterminé après transformation dans le domaine temporel (option disponible sur l'ARVM du laboratoire).

\subsubsection{Mesures en mode de transmission}

En tenant compte de toutes les composantes d'incertitudes [16] liées au calibrage de l'analyseur et à la mesure du facteur de transmission d'un composant sous test, en module et en phase, le bilan d'incertitudes peut être dressé de la manière suivante :

\subsubsection{Budget d'incertitudes en mode de transmission (amplitude) [17]}

D'après le tableau 2, l'incertitude combinée sur l'amplitude du paramètre de transmission, $u\left(\left|S_{21}\right|\right)$, est $0,016 \mathrm{~dB}$.

\subsubsection{Expression de l'évaluation de l'incertitude combinée, exprimée en degré, en mode de transmission (phase) [17]}

$$
\begin{aligned}
u\left(\angle S_{21}\right)= & \frac{180}{\pi} \arcsin \left(10^{\frac{u\left(\left|S_{21}\right|\right)}{20}}-1\right)+K \cdot f \\
& +1,2 \cdot u(L) \cdot f .
\end{aligned}
$$

- Le premier terme correspond à la contribution liée à l'incertitude de la mesure de transmission :

$$
\frac{180}{\pi} \arcsin \left(10^{\frac{u\left(\left|S_{21}\right|\right)}{20}}-1\right)=0,11^{\circ}\left(1,9 \times 10^{-3} \mathrm{rad}\right),
$$

où $u\left(\left|S_{21}\right|\right)$ est l'incertitude combinée, exprimée en décibel, développée dans le tableau 2.

- Le deuxième terme tient compte de l'incertitude sur la flexion de deux câbles :

$$
K \cdot f=0,70^{\circ}\left(12,2 \times 10^{-3} \mathrm{rad}\right),
$$

avec une fréquence d'opération $f=14 \mathrm{GHz}$ et une valeur de flexion pour les deux câbles de haute performance de $K=2 \times 0,025^{\circ} / \mathrm{GHz}$.

- Le dernier terme tient compte de l'incertitude sur la phase de la ligne étalon :

$$
1,2 \cdot u(L) \cdot f=0,21^{\circ}\left(3,66 \times 10^{-3} \mathrm{rad}\right),
$$

avec une fréquence d'opération $f=14 \mathrm{GHz}$ et une valeur issue d'un certificat d'étalonnage pour une ligne, soit $0,025 \mathrm{~mm}: u(L)=0,0125 \mathrm{~mm}$.

Soit, une incertitude combinée sur la phase du paramètre de transmission :

$$
u\left(\angle S_{21}\right)=1,02^{\circ}\left(17,80 \times 10^{-3} \mathrm{rad}\right)
$$

\subsubsection{Détermination du retard de groupe}

Le retard de groupe, exprimé en seconde, est déterminé à partir de la relation suivante :

$$
\tau=-\frac{1}{360} \times \frac{d}{d f}\left[\angle S_{21}\right],
$$


Tableau 3

Budget d'incertitudes de mesure de l'amplitude du paramètre $S_{11}(0,005)$.

\begin{tabular}{|l|c|c|c|c|}
\hline \multicolumn{1}{|c|}{ Contribution } & Incertitude & Distribution & Division & Incertitude type \\
\hline $\mathrm{B}_{1}:$ directivité et désadaptation effective & 0,00812 & $U$-shaped & $1,414,2$ & 0,00574 \\
\hline $\mathrm{B}_{2}:$ déséquilibre entre voies & 0,00001 & Rectangulaire & 1,7321 & 0,00001 \\
\hline $\mathrm{B}_{3}:$ linéarité & 0,00005 & Rectangulaire & 1,7321 & 0,00003 \\
\hline $\mathrm{B}_{4}:$ flexion du câble & 0,00002 & Gaussienne & 2 & 0,00001 \\
\hline $\mathrm{B}_{5}:$ conditions ambiantes & 0,00001 & Rectangulaire & 1,7321 & 0,00001 \\
\hline $\mathrm{A}_{1}:$ répétabilité du système & 0,00005 & Gaussienne & 2 & 0,00003 \\
\hline $\mathrm{A}_{2}:$ répétabilité du connecteur & 0,00500 & Gaussienne & 2 & 0,00250 \\
\hline Incertitude combinée, $\boldsymbol{u}\left|\boldsymbol{S}_{\mathbf{1 1}}\right|$ & & & & $\mathbf{0 , 0 0 6} \mathbf{2 6}$ \\
\hline
\end{tabular}

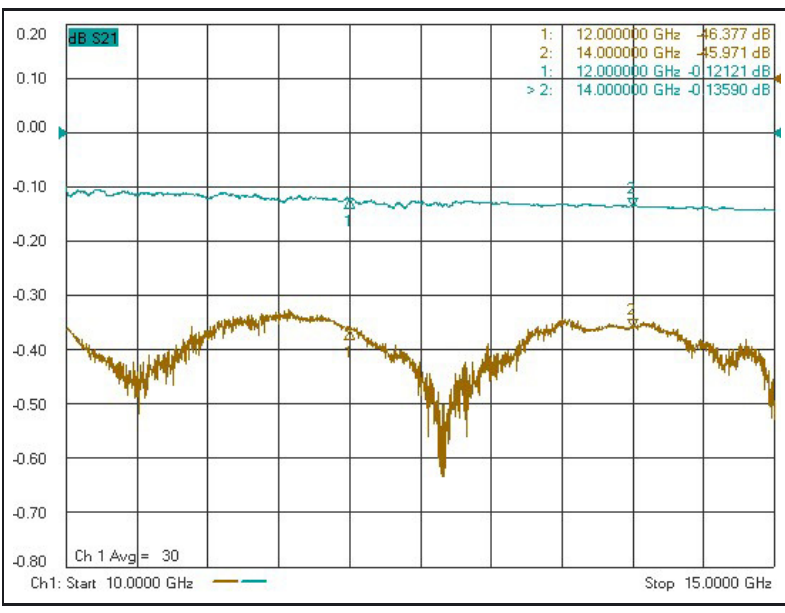

Fig. 10. - Mesure des facteurs de réflexion et de transmission, en amplitude, sur une ligne $50 \Omega$.

où $d f$ est l'ouverture de fréquence, exprimé en hertz, sur laquelle le retard est déterminé par rapport à la différence de phase, exprimée en degré, du paramètre de transmission.

Application numérique : pour une ouverture de fréquence $\Delta f \approx 3 \mathrm{MHz}, u(\tau) \approx 200 \mathrm{ps}$.

\subsubsection{Validation de la méthode}

Elle est faite par la caractérisation d'une ligne à air étalon de longueur et d'impédance connues.

Une ligne à air $50 \Omega$ de longueur $75 \mathrm{~mm}$, extraite du kit de vérification Agilent 85053B a été mesurée en amplitude (Fig. 10), en phase et en retard de groupe (Fig. 11).

Sur toute la bande de fréquence considérée $(10 \mathrm{GHz}-$ $15 \mathrm{GHz}$ ), les pertes de la ligne varient entre $0,10 \mathrm{~dB}$ et $0,15 \mathrm{~dB}$, en fonction de la fréquence, le facteur de réflexion restant inférieur à $45 \mathrm{~dB}$. Les valeurs obtenues sont comparables, aux incertitudes près, à celles figurant dans le certificat d'étalonnage correspondant [18].

D'après la figure 11, la valeur moyenne du retard de groupe mesuré est de 252,247 ps $\pm 4,192$ ps. Par comparaison, la valeur théorique du retard de la ligne à air $\left(\varepsilon_{\mathrm{r}}=1, c=299792458 \mathrm{~m} / \mathrm{s}\right)$ est de $250 \mathrm{ps}$, soit environ 2 ps d'écart entre les deux valeurs, résultat obtenu tout

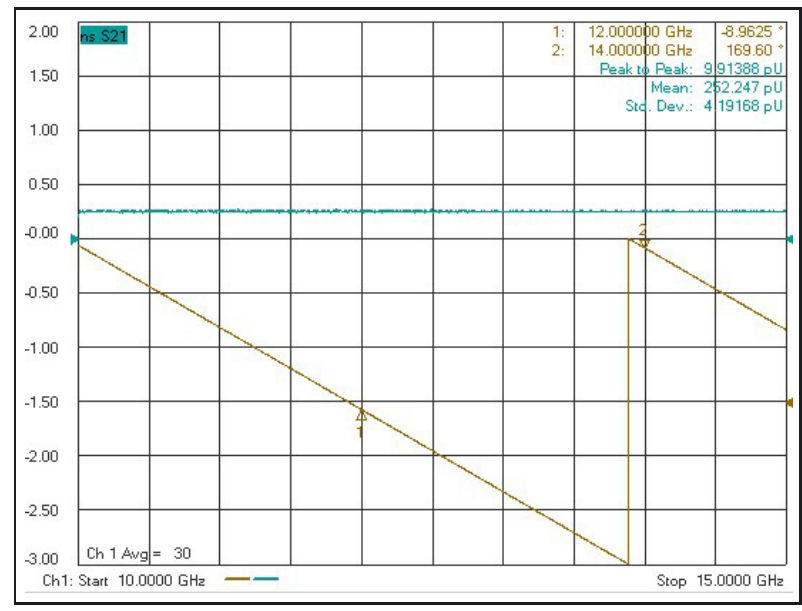

Fig. 11. - Mesure de la phase du facteur de transmission et du retard de groupe sur une ligne $50 \Omega$.

à fait excellent. Notons toutefois, que la valeur faible de l'écart type a pu être obtenue à l'aide de la fonction de lissage utilisée sur l'analyseur. En désactivant cette fonction, l'écart type de mesure est de l'ordre de 70 ps.

\subsubsection{Mesures en mode de réflexion}

En tenant compte de toutes les composantes d'incertitudes [16] liées à la fois au calibrage de l'analyseur et des mesures de paramètres de réflexion, en module et en phase, le bilan d'incertitudes peut être dressé de la manière suivante :

\subsubsection{Budget d'incertitudes en mode de réflexion (amplitude) [17]}

D'après le tableau 3, l'incertitude combinée sur l'amplitude du paramètre de réflexion, $u\left(\left|S_{11}\right|\right)$, est 0,006 3 .

\subsubsection{Détermination du retard de propagation dans le domaine temporel}

L'élément sous test étant court-circuité (élément à un accès), le passage dans le domaine temporel permet de déterminer le retard de propagation aller - retour à partir de la mesure du facteur de réflexion $S_{11}$. 


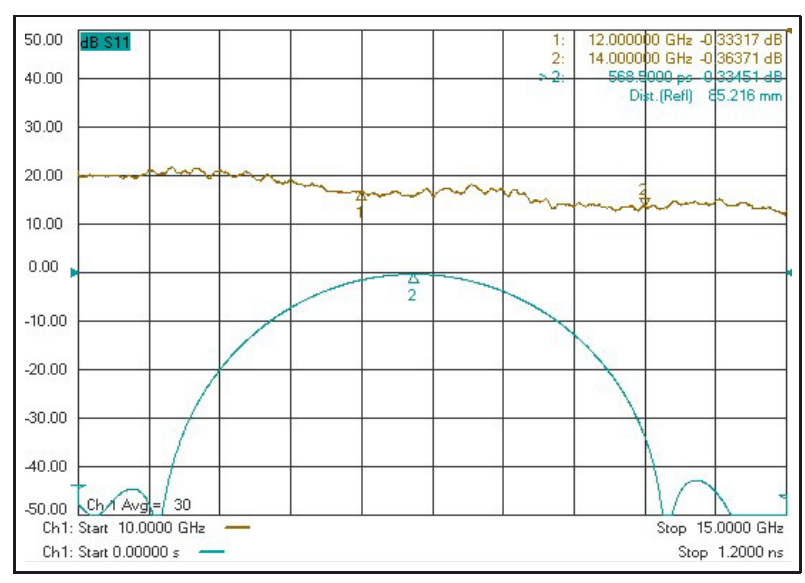

Fig. 12. - Mesure de l'amplitude du facteur de réflexion (domaine fréquentiel) et du retard associé (domaine temporel) sur une ligne $50 \Omega$ court-circuitée.

Ce passage dans le domaine temporel s'effectue par transformée de Fourier inverse en utilisant la technique de transformée de Fourier rapide Chirp-Z [13]. Le développement de cette partie dépasse l'objet de cet article. Cependant, nous présentons les résultats de mesures effectuées avec cette méthode et validées par comparaison avec la méthode de mesure en mode de transmission.

\subsubsection{Validation de la méthode}

Elle est faite par la caractérisation d'une ligne à air étalon court-circuitée, de longueur et d'impédance connues.

La même ligne à air est utilisée, associée à un court circuit extrait du kit de calibrage Agilent 85052C. Ce court circuit ayant servi au calibrage de l'analyseur en tant qu'élément inconnu à fort facteur de réflexion, sa caractérisation est nécessaire pour la validation de la méthode. N'ayant pas une longueur nulle, il présente un retard de 31,785 ps d'après le kit de calibrage. En appliquant la méthode décrite dans ce paragraphe, le retard de groupe mesuré est de 63,750 ps (aller - retour), soit un retard de 31,875 ps. Par comparaison, un écart de 90 fs est obtenu entre la valeur mesurée et la valeur extraite du kit de calibrage.

Sur la figure 12, sont reportées les mesures du facteur de réflexion et du retard associé de la ligne à air courtcircuitée.

Les pertes sur la ligne varient entre $0,3 \mathrm{~dB}$ et $0,4 \mathrm{~dB}$ sur toute la bande de fréquence. Le retard obtenu, après transformation dans le domaine temporel, est de 568,500 ps, soit un retard de la ligne à air de 252,375 ps, après élimination du retard du court circuit. Ainsi, l'écart de mesure du retard de la ligne à air, en utilisant les deux méthodes décrites ci-dessus, est de 128 fs, résultat obtenu tout à fait exceptionnel.

Pour conclure, l'analyseur de réseau vectoriel constitue un outil très performant pour mesurer le retard de groupe de tout élément, linéaire et non linéaire, aux

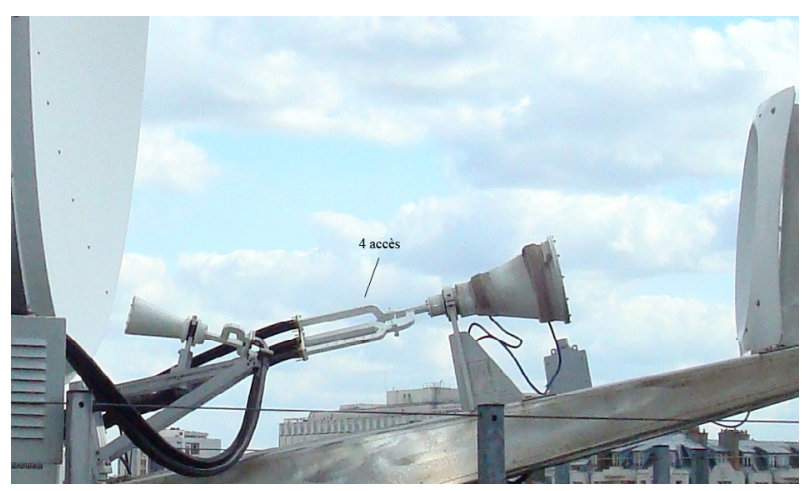

Fig. 13. - Vue rapprochée des deux cornets dont l'un est équipé d'un diplexeur à quatre accès.

fréquences d'opération. Cette mesure trouvera tout son sens par une évaluation rigoureuse de toutes les composantes d'incertitudes liées à cette mesure [23]. Des précautions particulières de mesure de retard accompagnées d'une grande qualité de l'élément sous test, permettent d'atteindre une incertitude combinée de quelques centaines de picosecondes aux fréquences micro-ondes. En revanche, des incertitudes dégradées qui peuvent atteindre une nanoseconde doivent être appliquées sur la mesure de retard de câbles de performances électromagnétiques quelconques.

\section{Applications particulières du simulateur de satellite}

Au-delà de sa fonction principale de détermination du retard différentiel de la station deux voies et de sa variation dans le temps, le simulateur de satellite joue un rôle important lors de changement de configuration d'équipement dans la station deux voies ; c'est le cas en 2008 lors d'un changement de satellite (passage de Intelsat IS-707 à IS-3R) accompagné d'une modification du module d'excitation du cornet d'antennes entraînant un changement du diplexeur à deux accès par un diplexeur à quatre accès (Fig. 13).

En effet, les nouvelles fréquences micro-ondes fournies par l'opérateur de satellite INTELSAT étaient polarisées orthogonalement à l'émission (comme à la réception), entre l'Europe et les États-Unis. De ce fait, deux solutions étaient possibles : abandonner un des deux réseaux (Europe ou États-Unis) ou changer de diplexeur. Evidemment, c'est la deuxième solution qui a été retenue par le laboratoire.

Ainsi, grâce à l'utilisation des deux stations deux voies du laboratoire, judicieusement installées (Fig. 3) de manière à pouvoir les positionner en mode de colocation, du simulateur de satellite équipant l'une des stations et de l'analyseur de réseau vectoriel micro-ondes, la continuité du lien étalonné UTC(OP)-UTC(PTB), pour les besoins du TAI, a pu être assuré avec le même niveau d'incertitude ( $\sim 1 \mathrm{~ns})$. Ce qui s'est traduit par une non dégradation de l'incertitude combinée sur l'écart UTC-UTC(OP) qui est publié, mensuellement, dans la circulaire T du BIPM, comme il est illustré sur la figure 14. 


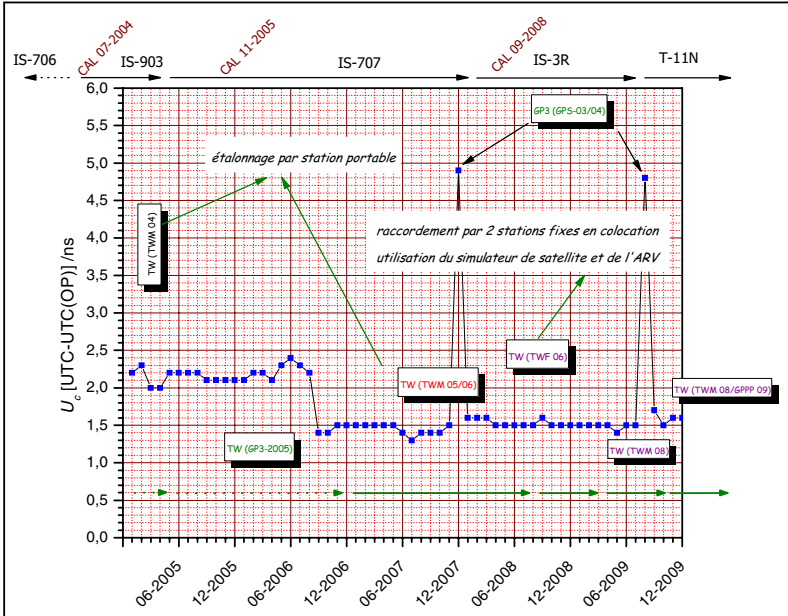

Fig. 14. - Historique de l'incertitude combinée sur l'écart UTC-UTC(OP) publiée par le BIPM.

Sur la figure 14 est reporté l'historique de l'incertitude combinée sur l'écart UTC-UTC(OP) issu des publications du BIPM couvrant la période de janvier 2005 à décembre 2009. Pendant cette période, il y a eu trois changements de satellite et trois étalonnages ([19-21]) par station portable $(2004,2005$ et 2008), le lien au TAI a été assuré entièrement par TWSTFT, excepté deux mois : celui de décembre 2007 où le GPS P3 a pris le relais (avec une dégradation nette de l'incertitude, soit $\sim 5 \mathrm{~ns}$ ), suite à une absence de données deux voies pendant une dizaine de jours (du 25 décembre au 2 janvier) liée à une coupure électrique survenu sur le site de l'Observatoire de Paris ayant engendré un disfonctionnement de la station deux voies, et celui d'août 2009, suite au dernier changement de satellite où les valeurs d'étalonnage des liens corrigées par la méthode GPS n'étaient pas encore disponibles [22]. Ainsi, le maintien de l'incertitude combinée globalement dans l'intervalle $[1,5-1,6]$ ns est clairement montré, à partir de février 2008, date de changement de satellite et de modification du système d'antenne sur la station OP01 du laboratoire.

D'autre part, il a été constaté que lors des campagnes d'étalonnage par station portable, les fluctuations de bruit mesurées en mode de colocation (OP01, TUG03) peuvent atteindre la valeur de 2,5 ns sur un jour de mesure par site visité, comme il est montré sur la figure 15 (données extraites de la campagne d'étalonnage 2008). La même amplitude de fluctuations a été observée également dans les autres sites européens visités par la station portable TUG03 [20].

Afin de comprendre et d'expliquer d'une part cette grande amplitude obtenue, ayant une influence directe sur l'incertitude d'étalonnage, ainsi que les sauts consécutifs observés propres au site de l'Observatoire de Paris (équipement compris), des mesures spécifiques ont été effectuées au laboratoire en utilisant la même horloge, les deux stations deux voies disponibles (OP01, OP02) et en appliquant le même programme d'étalonnage mais sur une durée de mesure plus longue (environ deux jours).

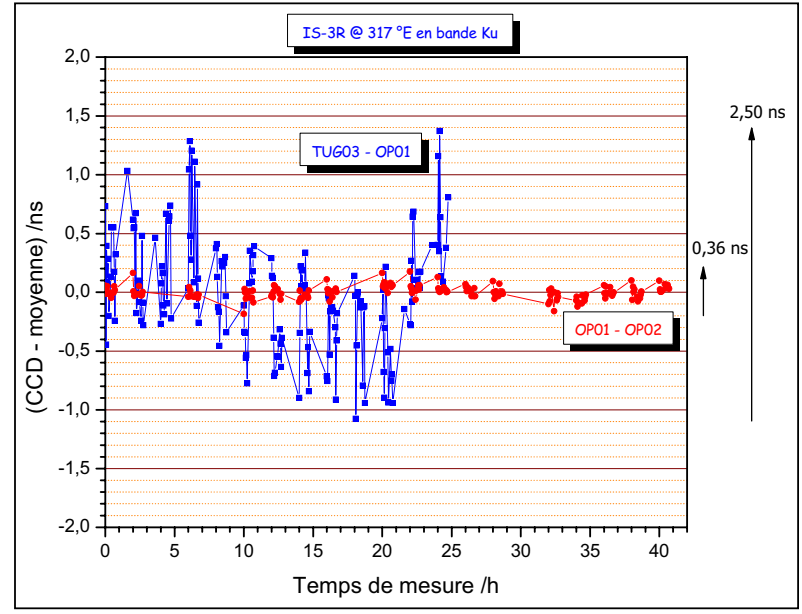

Fig. 15. - Mesures en colocation effectuées sur le site de l'Observatoire de Paris.

Le résultat obtenu (Fig. 15) est excellent et pertinent : les fluctuations de bruit sont inférieures à 200 ps en supprimant quelques outliers). Ceci met en évidence le fait qu'une station portable de performances moyennes et/ou moyennement adaptée aux sites visités (cas du site de l'Observatoire de Paris en particulier) dégrade les performances globales de l'étalonnage par une augmentation des fluctuations de bruit.

D'après la dernière expérience effectuée au laboratoire, on démontre que l'incertitude combinée d'étalonnage, en relatif, des retards différentiels des stations deux voies peut atteindre une valeur inférieure à la nanoseconde, si et seulement si les conditions suivantes sont respectées :

- station fixe de hautes performances électromagnétiques (adaptation, pertes, bruit, rendement);

- station fixe équipée d'un simulateur de satellite ayant un bruit résiduel global très faible (quelques dizaines de picoseconde);

- station portable de performances équivalentes à la station fixe.

\section{Conclusion et perspectives}

Un simulateur de satellite, de conception originale, destiné principalement à la caractérisation du retard différentiel, et de sa variation dans le temps, de la station terrienne deux voies du laboratoire a été développé. L'aspect original repose sur le concept de mesure qu'il fournit avec seulement deux configurations d'état et l'utilisation d'un analyseur de réseau vectoriel pour la détermination de ses retards internes.

La détermination de ce retard différentiel (et de sa variation) est indispensable dans le cas de comparaisons d'échelles de temps entre horloges distantes, car il s'agit d'un paramètre intrinsèque de l'équation horaire par liaison satellitaire micro-ondes à deux voies. Bien que ce paramètre soit souvent déterminé, de façon relative (par 
rapport au retard équivalent dans la station portable), dans le cadre de campagne d'étalonnage ponctuelle par station portable, le simulateur de satellite mesure en permanence l'évolution de ce retard dans le temps et en tient compte, en temps réel, dans l'équation horaire ce qui a pour conséquence une amélioration constante des incertitudes de mesure.

L'expérience développée au laboratoire et présentée dans cet article démontre que l'on peut atteindre une incertitude combinée de 0,4 ns sur la mesure du retard différentiel absolu d'une station terrienne en utilisant un simulateur de satellite tandis que les campagnes d'étalonnage conduites à ce jour démontrent qu'une incertitude combinée d'au moins 1 ns est obtenue sur la mesure du retard différentiel relatif entre deux stations terriennes.

Le simulateur de satellite est et demeure toujours d'une grande utilité lorsque pour une raison donnée (amélioration du fonctionnement de la station par rajouts de dispositifs complémentaires de mesure, d'adaptation ou de contrôle, défaillance d'un composant, etc.), une modification matérielle doit être effectuée sur la station terrienne étalonnée : grâce aux mesures effectuées par le simulateur, une correction peut être apportée à l'équation horaire sans dégradation significative (à 100 ps près) de l'incertitude d'étalonnage.

Dans les perspectives, un fonctionnement permanent du simulateur à plus long terme, sur une période d'un an et plus permettra une analyse plus approfondie du comportement de la station terrienne (et des liaisons deux voies « étalonnées » associées) dans l'objectif continu de réduire davantage les incertitudes de mesure effectuées par la méthode deux voies.

D' autre part, le groupe de travail TWSTFT du CCTF restera sensible aux développements de simulateurs de satellite dans les laboratoires. À part la commercialisation très limitée par Timetech d'un seul type de simulateur basé sur un ancien et premier modèle développé conjointement par le VSL et TUG, seul le VSL travaille aujourd'hui sur une nouvelle conception de simulateur dont il sera à l'avenir intéressant de comparer les performances sur le lien OP-VSL par exemple. Le BIPM encourage les laboratoires qui développent des simulateurs de satellite à démontrer le réel bénéfice qui peut être obtenu sur des liens opérationnels TWSTFT participant au TAI afin que cela puisse être pris en compte ultérieurement dans les Circular T.

\section{Remerciements}

L'auteur remercie l'équipe métrologie du temps dans laquelle ce travail est effectué, en particulier, P. Merck (doctorant 2002-2005) ayant travaillé sur le simulateur de satellite et $\mathrm{D}$. Rovera pour sa contribution récente dans les étalonnages relatifs.

J'exprime ma profonde reconnaissance à Agilent Technologies France pour son support technique, en personnel et en matériel, dans les mesures de retard absolu de mélangeurs micro-ondes, ainsi qu'à D. Allal du LNE pour son soutien à travers la mise à disposition ponctuelle des moyens de son laboratoire.
L'auteur remercie également les membres du groupe de travail TWSTFT du CCTF, notamment pour l'organisation de campagnes d'étalonnage en relatif par station portable.

\section{Références}

[1] DE JoNG G., "Delay stability of the TWSTFT earth station at VSL", EFTF, Warsaw 1998, 175-181.

[2] "The operational use of two-way satellite time and frequency transfer employing pseudorandom noise codes", Rec. ITU-R TF.1153-3, 2010.

[3] ACHKAR J., «Métrologie micro-ondes et métrologie $\mathrm{du}$ temps », Habilitation à diriger des recherches de l'Université Paris-6, avril 2004.

[4] MERCK P., «Développement d'une station terrienne de comparaisons d'horloges atomiques par liaisons microondes avec un satellite de télécommunications », Thèse de doctorat de l'Observatoire de Paris, décembre 2005.

[5] ACHKAR J. et MERCK P., « Comparaisons d'horloges atomiques au sol par liaisons micro-ondes deux voies avec un satellite de télécommunications ", Revue française de métrologie, 6, 2006, 9-24.

[6] MercK P. et ACHKAR J., "Design of a Ku band delay difference calibration device for TWSTFT station", CPEM, London, 2004.

[7] MercK P. et ACHKAR J., "Design of a Ku band delay difference calibration device for TWSTFT station", IEEE Instr. $\mathcal{G}$ Meas., 54, 2005, 814-818.

[8] ACHKAR J., "Development of a Ku band satellite simulator for TWSTFT applications", 21th EFTF, Genève, 2007.

[9] Merck P. et ACHKAR J., "Stability study of the TWSTFT microwave satellite simulator developed at LNE-SYRTE”, CPEM, Torino, 2006, 600-601.

[10] ACHKAR J., "A new microwave satellite simulator for the determination of delays in a TWSTFT station", 38th EuMC, Amsterdam, 2008.

[11] Piester D., Achkar J., Hlavac R., De Jong G., Blanzano B., Ressler H., Becker J., MercK P. et KOUDELKA O., "Calibration of four European TWSTFT earth stations with a portable station through INTELSAT 903", 19th EFTF, Besançon, mars 2005.

[12] ENGEN G.F., "Microwave circuit theory and foundations of microwave metrology", IEE Electrical Measurement Series 9, 1992.

[13] “Time domain analysis using a network analyzer", Agilent Application note 1287-12, 2007.

[14] D. SARIASLANI D. et DUNSMORE J., "Novel vector measurement technique for making fixed IF mixer measurements", Agilent Technologies document.

[15] ACHKAR J., "Design, realization and application of a satellite simulator in a TWSTFT station", 40th EuMC, Paris, 2010.

[16] "Guide to the expression of uncertainty in measurement", ISO/IEC Guide 98-3, 2008. 
[17] DE VREEDE J., "Draft proposed revision to the EA guidelines on evaluation of VNA to include uncertainty in phase”, ANAMET report 038, August 2003.

[18] "Certificate of calibration", Agilent certificate 85053B2815A0170820070606, 2007.

[19] PIESTER D. et al., "Calibration of six European TWSTFT earth stations using a portable station", 20th EFTF, Braunschweig, 2006, 460-467.

[20] Bauch A., Piester D., Whibberley P., Blanzano B., KoudelKa O., AchKar J., Rovera D., Kroon E., DIERIKX E., LORINI L., CORDARA F. et SCHLUNEGGER C., "Results of the 2008 TWSTFT calibration of seven European stations", 23th EFTF, Besançon, 2009.
[21] Bauch A., Piester D., Whibberley P., Blanzano B., KoudelKa O., AchKar J., Rovera D., Kroon E., DIERIKX E., LORINI L., CORDARA F. et SCHLUNEGGER C., "Results of the 2008 TWSTFT calibration of seven European stations", submitted for publication to the IEEE Instr. $\mathcal{E}$ Meas., 2009.

[22] Jiang Z., Lewandowski W. et Piester D., "Restoration the TWSTFT link calibration using GPS bridging after the satellite switch from IS-3R to T11-N in July / August 2009”, BIPM TM 166 report, 2009.

[23] "Guidelines on the evaluation of vector network analysers (VNA)", EURAMET/cg-12/v.01, 2007.

Article reçu le 15 janvier 2010, version révisée reçue le 18 novembre 2010. 